\title{
Analysis Traffic Volume of Rigid Pavement Damage on Roads Badami Karawang
}

\author{
Lutfi Abiansyah and Andri Irfan Rifai \\ Faculty of Engineering, University of Mercu Buana Jakarta, Indonesia \\ abiansyahlutfi@gmail.com, andrirfan@yahoo.com
}

\begin{abstract}
The road was a means of land transport is very important to the social relations and economic goods and services, and with a population that is increasing in every year to year, increasing the number of vehicles passing on the road that there is, therefore, in need planning a safe way according to the function, volume, and nature of traffic. Road repairs cost a lot and this action is felt not quite right because remedies can not survive by age plans and in kutif of IDN Times in 2019 that the condition damages the highway in the district of Karawang based on data from the Department of Public Works and Spatial Planning (PUPR) Khanewal district, along with the road status 1937.53 kilometers of district roads in poor condition. (IDN Times, 2019). This study was conducted to assess how much influence the volume of vehicles to the damage occurred on roads Badami karawang at STA $0+000-0+500$ and STA $2+250-2+750$, to examine the above problems by analyzing data from LHR and road damage the method of Highways, then further by looking at the correlation between the volume of vehicles on the road destruction by using SPSS and to increase the strength of rigid pavement using cement concrete road pavement planning 2003 is an additional layer (overlay) using the rigid pavement. From the analysis and discussion get Simultaneous Effect Hypothesis Test results that the Sig. $(0,000)<\alpha(0.05)$ and f count $(35.671)>$ F table (3.89) H0 is rejected. It can be concluded that the simultaneous effect of the Light vehicle road damage of $33.8 \%$, the simultaneous effect of the Heavy vehicle road damage amounted to $47.7 \%$, Motorcycles simultaneous effect on road damage by $12.1 \%$ and the simultaneous effect of Motor Vehicles not to road damage by $14.2 \%$, the result of the identification of the type and class of road damage indicates that the need for administration of additional layer (overlay) as a treatment for damage to roads, based on the analysis of the design used road repair method with a design life of 10 years, namely: (Overlay) Rigid Pavement (Rigid Pavement) as thick as $18 \mathrm{~cm}$.and of analysts earn fees at cost (overlay) Rigid Pavement (Rigid Pavement) Rp. 9,114,626,20 / segments.
\end{abstract}

\section{Keywords}

Analysis of traffic volume, pavement overlay rigid, correlation rigid traffic volume against damage.

\section{Introduction}

According to (IDN Times, 2019) Damage highway in the district of Karawang based on data from the Department of Public Works and Spatial Planning (PUPR) Karawang, along the road status 1937.53 kilometers of district roads in poor condition.

Damage to roads in the Falkirk area often become the public spotlight, usually people often submit complaints road damage through the social media accounts of his, so that the Department PUPR Karawang more serious in addressing the problem because the construction of the road was immediately felt by the people and for the district government pay more attention to quality improvement roads are often damaged before the age of the specified path. (IDN Times, 2019).

Therefore it is very important to perform preventive maintenance and assuming the above background, so I took the title of this thesis with the title Analysis of the volume of traffic on the road destruction rigid in Badami karawang roads. 

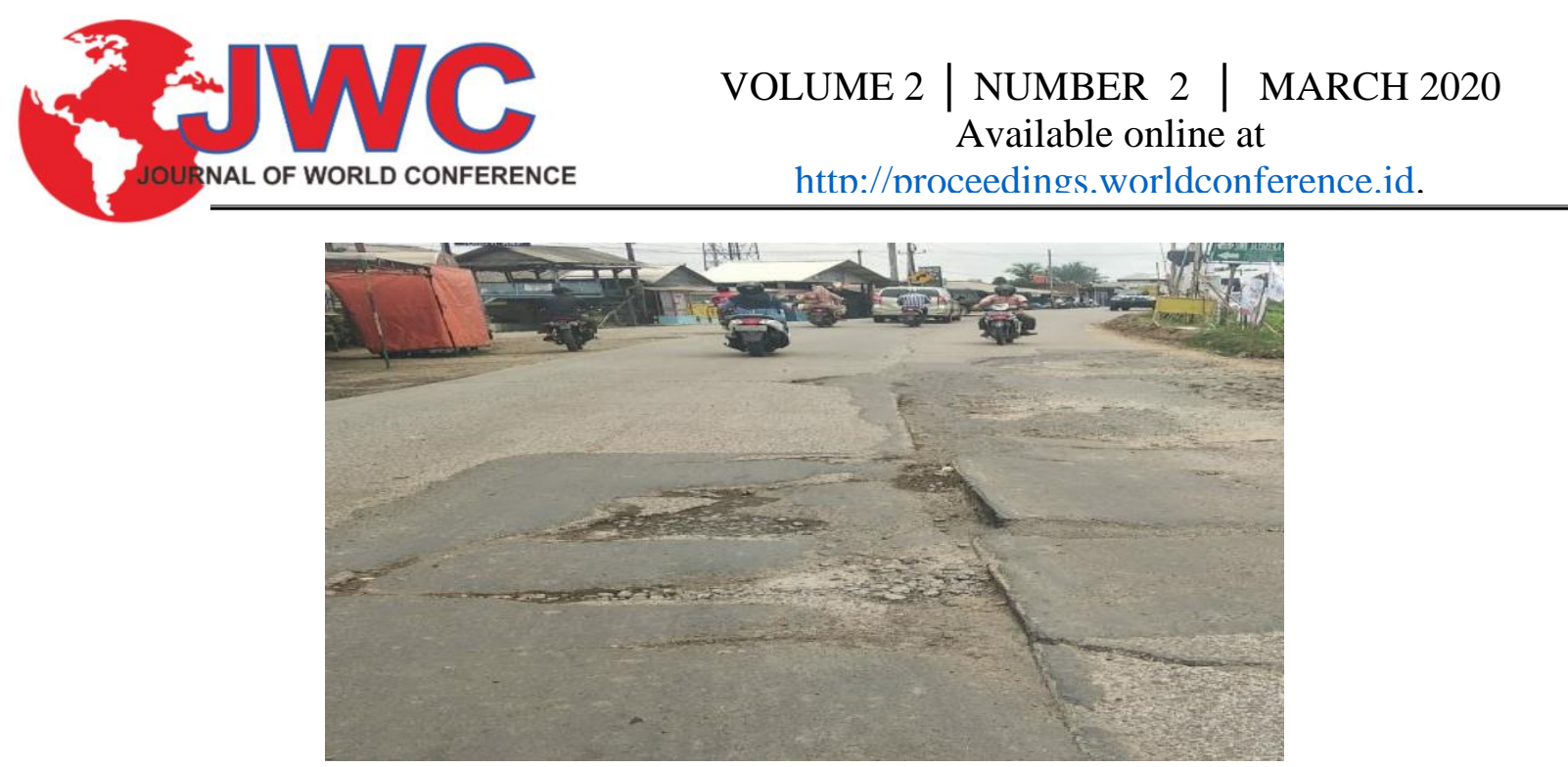

Source: radar karawang 2019

Figure 1. Damage to roads roads bedami karawang

Highway Badami is a street located in the district Telukjambe West, the road is an alternative way to Bekasi and connecting roads Subdistrict Telukjambe West sub-district base, it causes high levels of mobilization on the road section, and make the construction of pavement rigid damaged before age plan determined by keluahuhan citizens and road users that often lead to accidents, especially for two-wheeler riders (radar karawang, 2019).

Damage in general is the increased volume of traffic load, poor drainage systems, poor material properties pavement construction, climate, soil conditions are unstable, the planning of pavement is very thin, a job that does not deserve a process to the specification, this has happened because of the number of passing vehicles exceeding road capacity. Results damage reduction factor in the quality and age.

This study aims to Know the kind of damage that existed at the surface layer rigid in the streets Badami, determine the effect of the volume of vehicles with the level of damage to roads, know the methods of repair and how much it costs is needed, increase knowledge about the causes of road damage rigid due to the number of vehicles increasing increased. As well as provide new reference materials for civil engineering students and researchers, in order to increase knowledge about the causes of road damage caused by the increasing number of vehicles and can be used as a medium of teaching.

\section{Methodology}

Metodologogi research Overall research activities can be described as follows.

Method of collecting data.

1. Secondary data among other data required length and width of the road, the number of segments, the median, the number of lanes of the road and completeness.

2. Primary data volume of data traffic, road damage data

The analytical method used among others

1. Methods of analysis the volume of vehicles and Methods Using the Damage value of Highways.

2. Regression analysis method to obtain vehicle volume relationship patterns with the level of damage to roads.

3. Road repair method using cement concrete road pavement planning in 2003 to calculate the cement concrete slab thickness.

4. The cost of repair method, technique Hint unit price cost analysis of district road of 1995 to determine the total cost of road handling. 


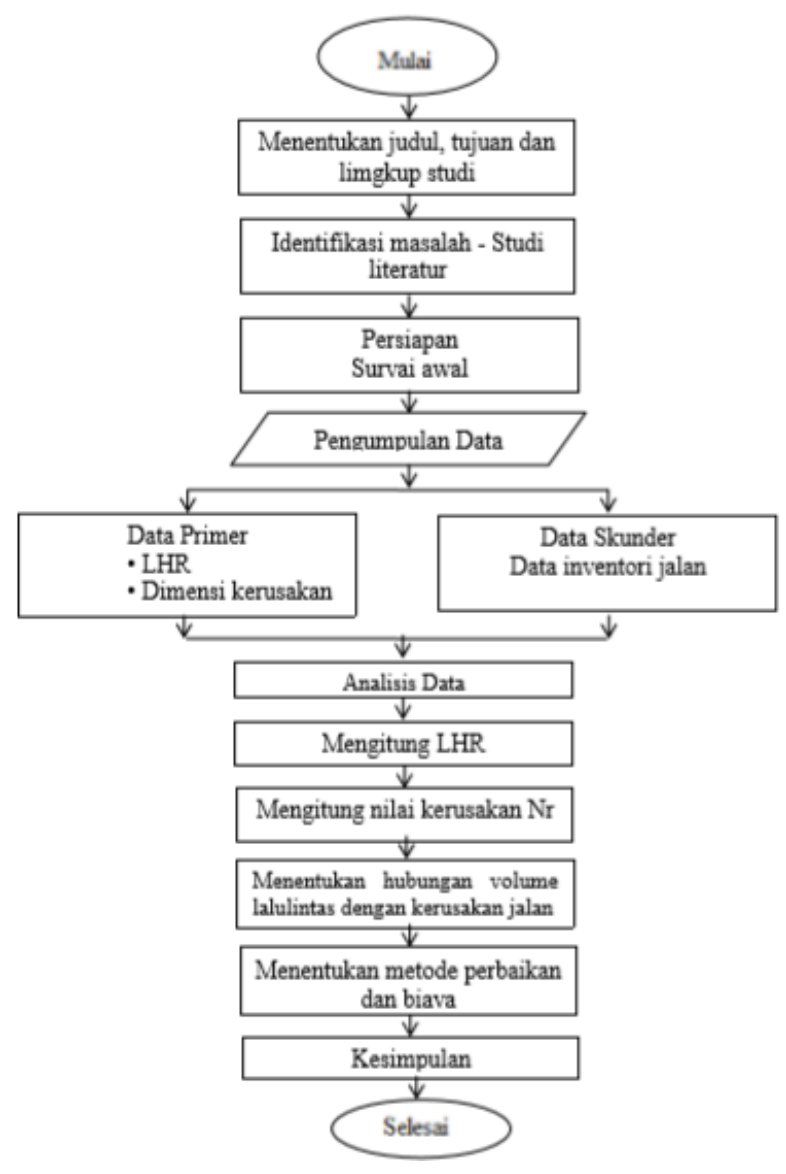

\section{Result}

\section{A. Traffic volume}

To capture data in the field within a week at peak hours beginning at 6:00 to 09:00 pm and 16:00 to 19:00 $\mathrm{pm}$, while the vehicle is observed light vehicle (LV), heavy vehicles (HV), Motorcycle (MC), vehicles are not motorized (UM).

Table 1. The volume of vehicle (veh / day)

\begin{tabular}{|c|c|c|c|c|c|c|}
\hline No. & Nama Jalan & Jalur & $\begin{array}{c}\text { Kendaraan } \\
\text { Ringan } \\
\text { (Kend/Hari) }\end{array}$ & $\begin{array}{c}\text { Kendaraan Berat } \\
\text { (Kend/Hari) }\end{array}$ & $\begin{array}{c}\text { Sepeda } \\
\text { Motor } \\
\text { (Kend/Hari) }\end{array}$ & $\begin{array}{l}\text { Kendaraa n } \\
\text { Tdk Bermotor } \\
\text { (Kend/Ha ri) }\end{array}$ \\
\hline \multirow{3}{*}{1} & \multirow{3}{*}{$\begin{array}{c}\text { badami - loji } \\
\text { STA . } 0+100-0+500\end{array}$} & & $($ (LV)) & $(\mathrm{HV})$ & (MC) & (UM) \\
\hline & & $\begin{array}{l}\text { Barat - } \\
\text { Timur }\end{array}$ & 2580 & 1811 & 14194 & 9 \\
\hline & & $\begin{array}{c}\text { Timur - } \\
\text { Barat }\end{array}$ & 1843 & 830 & 12631 & 6 \\
\hline \multirow{2}{*}{2} & \multirow{2}{*}{$\begin{array}{c}\text { badami - loji } \\
\text { STA .2+250- } 2+750\end{array}$} & $\begin{array}{l}\text { Barat - } \\
\text { Timur }\end{array}$ & 2480 & 1615 & 13650 & 7 \\
\hline & & $\begin{array}{c}\text { Timur - } \\
\text { Barat }\end{array}$ & 1432 & 930 & 11050 & 5 \\
\hline
\end{tabular}

Source: Data Analyst 


\section{B. Assess the damage}

From the observation or field observations obtained some kind of damage can occur: longitudinal cracks, transverse cracks, corner cracks, slippery Aggregates, Hole

Table 2. Sta $0+000-0+500$ Directions West - East

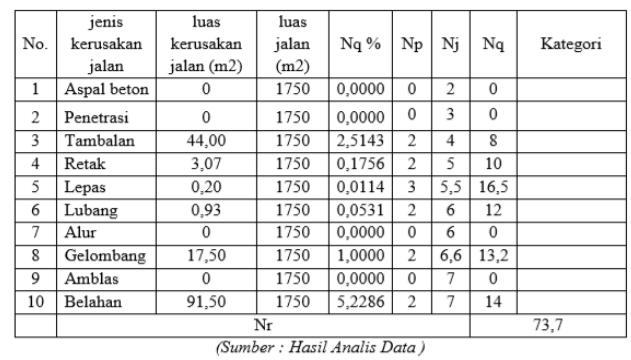

Table 4. Sta $2+250-2+750$ Directions West - East

\begin{tabular}{|c|l|c|c|c|c|c|c|c|}
\hline No. & $\begin{array}{c}\text { jenis } \\
\text { kerusakan } \\
\text { jalan }\end{array}$ & $\begin{array}{c}\text { luas } \\
\text { kerusakan } \\
\text { jalan }(\mathrm{m} 2)\end{array}$ & $\begin{array}{c}\text { luas } \\
\text { jalan } \\
(\mathrm{m} 2)\end{array}$ & $\mathrm{Nq} \%$ & $\mathrm{~Np}$ & $\mathrm{Nj}$ & $\mathrm{Nq}$ & Kategori \\
\hline 1 & Aspal beton & 0 & 1750 & 0,0000 & 0 & 2 & 0 & \\
\hline 2 & Penetrasi & 0 & 1750 & 0,0000 & 0 & 3 & 0 & \\
\hline 3 & Tambalan & 27,65 & 1750 & 1,5800 & 2 & 4 & 8 & \\
\hline 4 & Retak & 9,00 & 1750 & 0,5143 & 2 & 5 & 10 & \\
\hline 5 & Lepas & 0,25 & 1750 & 0,0143 & 2 & 5,5 & 11 & \\
\hline 6 & Lubang & 1,29 & 1750 & 0,0734 & 2 & 6 & 12 & \\
\hline 7 & Alur & 0 & 1750 & 0,0000 & 0 & 6 & 0 & \\
\hline 8 & Gelombang & - & 1750 & 0,0000 & 0 & 6,6 & 0 & \\
\hline 9 & Amblas & 0 & 1750 & 0,0000 & 0 & 7 & 0 & \\
\hline 10 & Belahan & 19,00 & 1750 & 1,0857 & 2 & 7 & 14 & \\
\hline & \multicolumn{7}{|c|}{ Nr } \\
\hline \multicolumn{70}{|c|}{ (Sumber : Hasil Analis Data) $)$} \\
\hline
\end{tabular}

Table 3. Sta $0+000-0+500$ Directions East - West

\begin{tabular}{|c|l|c|c|c|c|c|c|c|}
\hline No. & $\begin{array}{c}\text { jenis } \\
\text { kerusakan } \\
\text { jalan }\end{array}$ & $\begin{array}{c}\text { luas } \\
\text { kerusakan } \\
\text { jalan }(\mathrm{m} 2)\end{array}$ & $\begin{array}{c}\text { luas } \\
\text { jalan } \\
(\mathrm{m} 2)\end{array}$ & $\mathrm{Nq} \%$ & $\mathrm{~Np}$ & $\mathrm{~N}$ & $\mathrm{Nq}$ & Kategori \\
\hline 1 & $\begin{array}{l}\text { Aspal } \\
\text { beton }\end{array}$ & 0 & 1750 & 0,0000 & 0 & 2 & 0 & \\
\hline 2 & Penetrasi & 0 & 1750 & 0,0000 & 0 & 3 & 0 & \\
\hline 3 & Tambalan & 11,00 & 1750 & 0,6286 & 2 & 4 & 8 & \\
\hline 4 & Retak & 5,60 & 1750 & 0,3200 & 2 & 5 & 10 & \\
\hline 5 & Lepas & 0,10 & 1750 & 0,0057 & 2 & 5,5 & 11 & \\
\hline 6 & Lubang & 1,14 & 1750 & 0,0651 & 2 & 6 & 12 & \\
\hline 7 & Alur & 0 & 1750 & 0,0000 & 0 & 6 & 0 & \\
\hline 8 & Gelombang & 17,50 & 1750 & 1,0000 & 2 & 6,6 & 13,2 & \\
\hline 9 & Amblas & 0 & 1750 & 0,0000 & 0 & 7 & 0 & \\
\hline 10 & Belahan & 27,75 & 1750 & 1,5857 & 2 & 7 & 14 & \\
\hline \multicolumn{7}{|c|}{ Nr } \\
\hline \multicolumn{7}{|c|}{ (Sumber : Hasil Analis Data) $)$} \\
\hline
\end{tabular}

Table 5. Sta $2+250-2+750$ Directions East - West

\begin{tabular}{|c|l|c|c|c|c|c|c|c|}
\hline No. & $\begin{array}{c}\text { jenis } \\
\text { kerusakan } \\
\text { jalan }\end{array}$ & $\begin{array}{c}\text { luas } \\
\text { kerusakan } \\
\text { jalan }(\mathrm{m} 2)\end{array}$ & $\begin{array}{c}\text { luas } \\
\text { jalan } \\
(\mathrm{m} 2)\end{array}$ & $\mathrm{Nq} \%$ & $\mathrm{~Np}$ & $\mathrm{Nj}$ & $\mathrm{Nq}$ & Kategori \\
\hline 1 & Aspal beton & 0 & 1750 & 0,0000 & 0 & 2 & 0 & \\
\hline 2 & Penetrasi & 0 & 1750 & 0,0000 & 0 & 3 & 0 & \\
\hline 3 & Tambalan & 10,00 & 1750 & 0,5714 & 2 & 4 & 8 & \\
\hline 4 & Retak & 40,00 & 1750 & 2,2857 & 2 & 5 & 10 & \\
\hline 5 & Lepas & 0,10 & 1750 & 0,0057 & 2 & 5,5 & 11 & \\
\hline 6 & Lubang & 1,14 & 1750 & 0,0651 & 2 & 6 & 12 & \\
\hline 7 & Alur & 0 & 1750 & 0,0000 & 0 & 6 & 0 & \\
\hline 8 & Gelombang & 0 & 1750 & 0,0000 & 0 & 6,6 & 0 & \\
\hline 9 & Amblas & 0 & 1750 & 0,0000 & 0 & 7 & 0 & \\
\hline 10 & Belahan & 0 & 1750 & 0,0000 & 0 & 7 & 0 & \\
\hline & \multicolumn{7}{|c|}{$\mathrm{Nr}$} \\
\hline
\end{tabular}

\section{Determine the correlation of the volume of vehicles on the road destruction}

Analyzes were performed using SPSS. Recapitulation X1, X2, X3, X4 and Y. kendaraa volume and road damage were analyzed with SPSS non-linear regression. Variables that are used can be seen from the table below.

Table 6. Summary of variables $\mathrm{X}$ and $\mathrm{Y}$

\begin{tabular}{|c|c|c|c|c|}
\hline $\begin{array}{c}\text { Nilai } \\
\text { Kerusakan } \\
\text { Jalan }(\mathrm{Nr})(\mathrm{Y})\end{array}$ & $\begin{array}{c}\text { Kendaraan } \\
\text { Ringan } \\
(\text { Kend/Hari) } \\
(\mathrm{X} 1)\end{array}$ & $\begin{array}{c}\text { Kendaraan } \\
\text { Berat } \\
(\text { Kend/Hari) } \\
(\mathrm{X} 2)\end{array}$ & $\begin{array}{c}\text { Sepeda Motor } \\
(\text { Kend/Hari) } \\
(\mathrm{X} 3)\end{array}$ & $\begin{array}{c}\text { Kendaraa n Tdk } \\
\text { Bermotor } \\
(\text { Kend/Ha ri)(X4) }\end{array}$ \\
\hline 73,7 & 2580 & 1811 & 14194 & 9 \\
\hline 68,2 & 1792 & 830 & 12631 & 6 \\
\hline 55 & 2480 & 1615 & 13650 & 7 \\
\hline 41 & 1432 & 930 & 11050 & 5 \\
\hline
\end{tabular}

Source: Data Analyst 


\section{VOLUME 2 | NUMBER 2 | MARCH 2020}

Available online at

http://proceedings.worldconference.id.

Table 7.Result of data processing SPSS 16 Obtained coefficient for each variable track

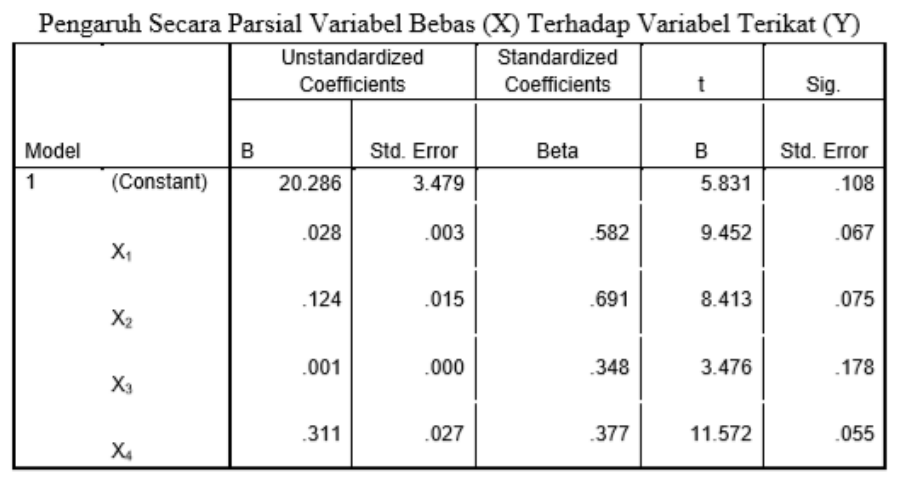

Source: Data Analyst

The effect can be concluded partial determination coefficient analysis of traffic volume mempegaruhi role in Karawang Badami road damage by light vehicles 33.8\%, 47.7\% Heavy Vehicles, Motorcycles $12.1 \%, 14.2 \%$ of non-motorized vehicles.

Table 8. Results of Testing the overall path analysis

\begin{tabular}{|l|r|r|r|r|}
\hline Koefisien Determinasi \\
\hline Model & \multicolumn{1}{|c|}{$\mathrm{R}$} & R Square & $\begin{array}{c}\text { Adjusted R } \\
\text { Square }\end{array}$ & $\begin{array}{c}\text { Std. Error of } \\
\text { the Estimate }\end{array}$ \\
\hline 1 & $.997(\mathrm{a})$ & .893 & .965 & 1.85797 \\
\hline
\end{tabular}

Source: Data Analyst

According to the table above shows that the coefficient of determination volume of vehicles on road damage 0.893 then this volume of vehicles has contributed damage 0.893 Street.

Table 9. Effect of Simultaneous Hypothesis Testing The volume of vehicles on the road destruction

\begin{tabular}{|c|c|c|c|c|c|}
\hline Structural & Sig. & A & F hitung & F tabel & Kesimpulan \\
\hline py $\mathrm{x}_{1} \mathrm{x}_{2}$ & 0,000 & 0,05 & 35,671 & 3,89 & Ho ditolak \\
\hline
\end{tabular}

Source: Data Analyst

According to the table above can be explained that the Sig. $(0,000)<\alpha(0.05)$ and $\mathrm{f}$ count $(35.671)>\mathrm{F}$ table (3.89) H0 is rejected. Thus dapatdisimpulkan that simultaneously affect traffic volume on road damage.

\section{Improved methods and costs}

1. Methods repair repair methods in use namely Structural Repair additional coating, the method of Highways ie additional coating rigid pavement with a design life of 10 years.

To calculate how thick the need for an additional layer by calculating:
a. calculate LHR
b. Analysts vehicle axis
c. Calculation repetition occurring axis
d. Calculation of the concrete slab thickness 


\section{a) Counting LHR}

In view of the value of the vehicle on a volume o b sta $0+000-0+500$ is greater than the $2+250-2+750$ can be seen in the table above, the sta $0+000-0+500$ to reference data to calculate how thick overlay (overlay) as a way of handling damage.

Light vehicle 2 ton $=4,372$ veh $/$ day / 2-way

Bus 8 ton $=420$ veh $/$ day / 2-way

Truck 2 as 13 tonne $=1,201$ veh $/$ day / 2 -way

Truck 3 as 20 ton $=1,020$ veh $/$ day / 2 -way

\section{b) Analysts vehicle axis}

For step - step analysis of the number of axes calculation is as follows. Determine the configuration of the load, determine the number of vehicles, determine the number of axes per vehicle, specify the number of axis $=$ number of vehicles $\mathrm{X}$ amount perkendaraan axis, determines the value of BS (axle) and JS (number of axes).

Table 10. Calculation of Total Axis By Type and His burden

\begin{tabular}{|c|c|c|c|c|c|c|c|c|c|c|c|c|c|}
\hline \multirow{2}{*}{$\begin{array}{l}\text { Jenis } \\
\text { Kend }\end{array}$} & \multicolumn{4}{|c|}{$\begin{array}{l}\text { Konfigurasi beban } \\
\text { sumbu (ton) }\end{array}$} & \multirow{2}{*}{$\begin{array}{l}\text { Jml } \\
\text { Kend } \\
\text { (bh) }\end{array}$} & \multirow{2}{*}{$\begin{array}{c}\text { Jml } \\
\text { Sumbu } \\
\text { per } \\
\text { Kend } \\
\text { (bh) }\end{array}$} & \multirow{2}{*}{$\begin{array}{c}\text { Jml } \\
\text { Sumbu } \\
\text { (bh) }\end{array}$} & \multicolumn{2}{|c|}{ STRT } & \multicolumn{2}{|c|}{ STRG } & \multicolumn{2}{|c|}{ STdRG } \\
\hline & $\begin{array}{l}\text { R } \\
\text { D }\end{array}$ & $\begin{array}{l}\mathrm{R} \\
\mathrm{B}\end{array}$ & $\begin{array}{r}\text { RG } \\
\text { D }\end{array}$ & $\begin{array}{c}\text { RG } \\
\text { B }\end{array}$ & & & & $\begin{array}{c}\text { BS } \\
\text { (ton) }\end{array}$ & $\begin{array}{l}\text { JS } \\
\text { (bh) }\end{array}$ & $\begin{array}{l}\text { BS } \\
\text { (ton) }\end{array}$ & $\begin{array}{l}\text { JS } \\
\text { (bh) }\end{array}$ & $\begin{array}{l}\text { BS } \\
\text { (ton) }\end{array}$ & $\begin{array}{l}\text { JS } \\
\text { (bh) }\end{array}$ \\
\hline (1) & \multicolumn{4}{|c|}{ (2) } & (3) & (4) & (5) & (6) & (7) & (8) & (9) & (10) & (11) \\
\hline MP & 1 & 1 & - & - & 4372 & - & - & - & - & . & - & - & - \\
\hline $\begin{array}{ll}\text { Bus } & 8 \\
\text { ton } & \end{array}$ & 3 & 5 & - & - & 420 & 2 & 840 & 3 & 420 & 5 & 420 & - & - \\
\hline Truk 2 as & 5 & 8 & - & - & 1201 & 2 & 2402 & 5 & 1201 & 8 & 1201 & - & - \\
\hline Truk 3 as & 6 & 14 & - & - & 1020 & 2 & 2040 & 6 & 1020 & - & - & 14 & 1020 \\
\hline \multicolumn{7}{|c|}{ Total } & 5282 & & 2641 & & 1621 & & 1020 \\
\hline
\end{tabular}

Source: Data Analyst

JSKN $=365 \times$ JSKNH $\times$ Rx C

$\mathrm{C}=0.5$

JSKN plan $=365 \times$ JSKNH $\times \mathrm{R} \times \mathrm{C}$

$=365 \times 5282 \times 13.18 \times 0.5$

$=12,392,530.68$ fruit. 


\section{c) Calculation repetition occurring axis}

Table 11. Repetition Axes Calculation Plan

\begin{tabular}{|c|c|c|c|c|c|c|}
\hline $\begin{array}{c}\text { Jenis } \\
\text { Sumbu }\end{array}$ & $\begin{array}{c}\text { Beban } \\
\text { Sumbu } \\
\text { (ton) }\end{array}$ & $\begin{array}{l}\text { Jumlah } \\
\text { Sumbu }\end{array}$ & $\begin{array}{c}\text { Proporsi } \\
\text { Beban }\end{array}$ & $\begin{array}{l}\text { Proporsi } \\
\text { Sumbu }\end{array}$ & $\begin{array}{l}\text { Lalu lintas } \\
\text { Rencana }\end{array}$ & Repetisi yang Terjadi \\
\hline-1 & -2 & -3 & -4 & -5 & -6 & $(7)=(4) \times(5) \times(6)$ \\
\hline \multirow{3}{*}{ STRT } & 6 & 2040 & 0,39 & 0,67 & $12.392 .530,68$ & $3.190 .806,84$ \\
\hline & 5 & 2402 & 0,45 & 0,67 & $12.392 .530,68$ & $3.757 .018,64$ \\
\hline & 3 & 840 & 0,16 & 0,67 & $12.392 .530,68$ & $1.313 .861,64$ \\
\hline \multicolumn{2}{|c|}{ Total } & 5282 & 1,00 & & & \\
\hline \multirow{2}{*}{ STRG } & 8 & 1201 & 0,74 & 0,20 & $12.392 .530,68$ & $1.878 .509,32$ \\
\hline & 5 & 420 & 0,26 & 0,20 & $12.392 .530,68$ & $656.930,82$ \\
\hline \multicolumn{2}{|c|}{ Total } & 1621 & 1,00 & & & \\
\hline STdRG & 14 & 1020 & 1 & 0,13 & $12.392 .530,68$ & $1.595 .403,42$ \\
\hline \multicolumn{2}{|c|}{ Total } & 1020 & 1 & & & \\
\hline \multicolumn{6}{|c|}{ Kumulatif } & $12.392 .530,68$ \\
\hline
\end{tabular}

Source: Data Analyst

\section{d) Calculation of the concrete slab thickness}

The data - the data used in the calculation of the concrete slab

1. $=10$ year design life.

2. Repetition is happening $=12,392,530.68$ fruit

3. Load safety factor $=1.1$

4. Flexural tensile strength $\left(f^{\prime} c f\right)=4 \mathrm{Mpa}$

5. Subgrade $\mathrm{CBR}=3.02 \%$.

6. Effective $\mathrm{CBR}=21.05 \%$ (obtained from the graph)

7. Estimates thick concrete $=25 \mathrm{~cm}$

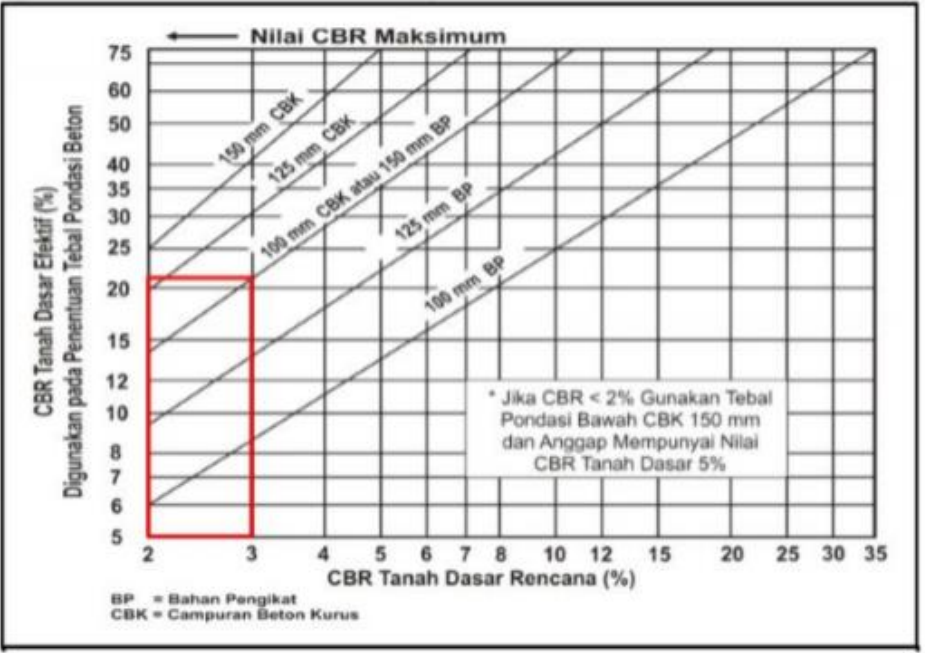

Source: Data Analyst

Figure 2. Relationship Graph graphic CBR CBR Effective Basic Land Basic Land Plan

After determining the thickness of the estimates on can then continue with the Table Voltage Equivalent and Voltage Erosion and in Table thick sleb 250 and values .cbr effective 20 in view the value of STRT, Strg, STRdRG then the value is applied to the graph TE (Voltage Equivalent) and FE (Erosion factor) To determine the estimated thick concrete slab if it is safe or not. 


\section{VOLUME 2 | NUMBER 2 | MARCH 2020}

Available online at

http://proceedings.worldconference.id.

Table 12. Fatigue Analysis and Erosion with estimates Thick Plates $25 \mathrm{~cm}$

\begin{tabular}{|c|c|c|c|c|c|c|c|c|c|}
\hline \multirow{2}{*}{$\begin{array}{c}\text { Jenis } \\
\text { Sumbu }\end{array}$} & \multirow{2}{*}{\multicolumn{2}{|c|}{$\begin{array}{c}\text { Beban Sumbu } \\
\text { (ton ke } \mathrm{kN} \text { ) }\end{array}$}} & \multirow{2}{*}{$\begin{array}{c}\text { Beban Rencana Per } \\
\text { Roda (kN) }\end{array}$} & \multirow{2}{*}{$\begin{array}{c}\text { Repetisi yang } \\
\text { Terjadi }\end{array}$} & \multirow{2}{*}{$\begin{array}{l}\text { Faktor } \\
\text { Tegangan } \\
\text { dari Erosi }\end{array}$} & \multicolumn{2}{|c|}{ Analisa Fatik } & \multicolumn{2}{|c|}{ Analisa Erosi } \\
\hline & & & & & & $\begin{array}{c}\text { Repetisi } \\
\text { Ijin }\end{array}$ & $\begin{array}{c}\text { Persen Rusak } \\
(\%)\end{array}$ & Repetisi Ijin & $\begin{array}{c}\text { Persen Rusak } \\
\text { (\%) }\end{array}$ \\
\hline-1 & \multicolumn{2}{|c|}{-2} & $\begin{array}{c}(3)=(2)^{*} \mathrm{FKB} / \\
\mathrm{jml} \text { roda }\end{array}$ & -4 & -5 & -6 & $\begin{array}{c}(7)= \\
(4)^{*} 100 /(6)\end{array}$ & -8 & $\begin{array}{c}(9)= \\
(4)^{*} 100 /(8)\end{array}$ \\
\hline \multirow[t]{3}{*}{ STRT } & 6 & 60 & 33 & $3.190 .806,84$ & $\mathrm{TE}=0,65$ & TT & 0 & TT & 0 \\
\hline & 5 & 50 & 27,5 & $3.757 .018,64$ & $\mathrm{FRT}=0,16$ & TT & 0 & TT & 0 \\
\hline & 3 & 30 & 16,5 & $1.313 .861,64$ & $\mathrm{FE}=1,53$ & TT & 0 & TT & 0 \\
\hline \multirow[t]{3}{*}{ STRG } & 8 & 80 & 22 & $1.878 .509,32$ & $\mathrm{TE}=1,02$ & TT & 0 & TT & 0 \\
\hline & 5 & 50 & 13,75 & $656.930,82$ & $\mathrm{FRT}=0,26$ & TT & 0 & TT & 0 \\
\hline & - & - & - & - & $\mathrm{FE}=2,13$ & - & - & - & - \\
\hline \multirow[t]{3}{*}{ STdRG } & 14 & 140 & 19,25 & $1.595 .403,42$ & $\mathrm{TE}=0,87$ & TT & 0 & $2,00, \mathrm{E}+07$ & 0,08 \\
\hline & - & - & - & - & FRT $=0,22$ & - & - & - & - \\
\hline & - & - & - & - & $\mathrm{FE}=2,23$ & - & - & - & - \\
\hline \multicolumn{6}{|c|}{ Total } & \multicolumn{2}{|c|}{$0<100 \%$} & \multicolumn{2}{|c|}{$0,08<100 \%$} \\
\hline
\end{tabular}

Source: Data Analyst

Because the percentage of fatigue damage and erosion damage percentage to 0.08 and smaller than $100 \%$, the plate thickness used is $25 \mathrm{~cm}$ due to already meet the requirements of a strong and safe to use.

Determining the required thick layer added to the equation:

a. Determining thick layer immediately added:

Thick layers of added directly required $(\operatorname{Tr}) \operatorname{Tr}=1.4 \sqrt{ }(\mathrm{T} 1.4$-Cs.T0 1.4)

With known: $\mathrm{T}=$ thick layers needed $=25 \mathrm{~cm}$

$$
\mathrm{T} 0=30 \mathrm{~cm}
$$

$\mathrm{Cs}=0.35$

Then $\operatorname{Tr}=1.4 \sqrt{ }\{(25) 1.4-0.35(30) 1.4\}=8.81 \mathrm{~cm}$.

b. Determining the layer thickness plus a separator:

$\operatorname{Tr}=\sqrt{ }(\mathrm{T} 2-$ Cs.T0 2)

With note: $\mathrm{T} 0=30 \mathrm{~cm}$

Cs $=0.35$ (fatherly broken pavement conditions strruktur)

Then $\operatorname{Tr}=\sqrt{ }\{(25)$ from 2 to 0.35 (30) 2$\}=17.6 \mathrm{~cm}$

Taken thick layer added $\operatorname{Tr}=17.6 \mathrm{~cm}>\operatorname{Tr}$ minimum $=8.81 \mathrm{~cm}$

Then take a thick layer added $\neq \operatorname{Tr}=17.6 \mathrm{~cm} 18 \mathrm{~cm}$

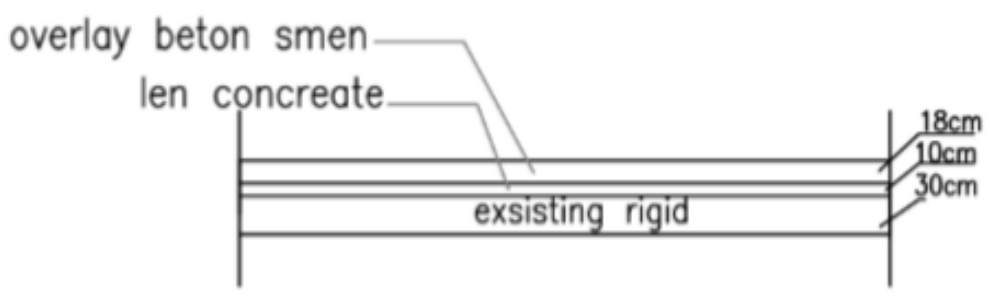

Source: Data Analyst

\section{Repair Cost}

A. The volume of work

B. Calculation of cost analysis of rigid pavement work

C. Total cost of road repairs per segment 


\section{A. The volume of work}

a. The width of the track

$=2 \times 3.5 \mathrm{~m}$

$=7 \mathrm{~m}$.

b. Long road

$=1000 \mathrm{~m}$.

c. The width of the segment

d. Segment length

$=3.5 \mathrm{~m}$.

e. Plate thickness

f. Cast Volume 1 segment

$=5 \mathrm{~m}$

$=0.18 \mathrm{~m}$.

$=\mathrm{LxWxH}$

$=5 \times 3.5 \times 0.18$

$=3.15 \mathrm{~m} 3$.

g.Volume Lean Wet Concrete

$=\mathrm{LxWxH}$

$=5 \times 3.5 \times 0.1$

$=1.75 \mathrm{~m} 3$

h. The number of segments required $=($ road length $/$ segment length $) \times 2$

$=(1000 / 5) \times 2$

$=400$ segments

\section{B. calculation of rigid pavement overlay HSP analyst}
a. Wet
b. HSP Wet Lean Concrete
c. HSP casting plate
d. Biaya Lean Concrete

e. Slab casting costs street

$$
\begin{aligned}
& =10 \text { year design life. } \\
& =\text { Rp. } 827,947.67 \\
& =\text { Rp. } 2,443,085.01 \\
& =\text { volume } x \text { unit price } \\
& =1.75, \text { x Rp. } 827,947.67 \\
& =\text { Rp. } 1,448,908.42 \\
& =\text { volume } x \text { unit price } \\
& =3.15 \times \text { Rp. } 2,443,085.01 \\
& =\text { Rp. } 7.695 .717 .78
\end{aligned}
$$

\section{Total cost of road repairs per segment \\ a. Cost Lean Wet Concrete = Rp. 1,448,908.42 \\ b. Cost casting plate = Rp. 7,695,717.78+ amount \\ $=$ Rp. 9,114,626.20} segments

So the total cost required to repair roads using additional layers of rigid pavement is Rp. 9,114,626.20 /

\section{Conclussion}

After analysis and discussion, it can be concluded:

1. Based on the results of Simultaneous Effect Hypothesis that the Sig. $(0,000)<\alpha(0.05)$ and $\mathrm{f}$ count (35.671)> F table (3.89) H0 is rejected. It can be concluded that the simultaneous effect of the Light Vehicle Damage to Roads of $33.8 \%$, the simultaneous effect of the Heavy Vehicle Road Damage amounted to $47.7 \%$, Motorcycles simultaneous effect against 12.1\% Damage Roads and Motor Vehicles no simultaneous effect against Road damage by $14.2 \%$..

2. Based on analysis of the design used road repair method with a design life of 10 years, namely: Rigid Pavement Overlay (Rigid Pavement) as thick as $18 \mathrm{~cm}$.

3. Based on analysis of the cost calculation of the fees obtained by the third method of repair is equal to: the cost of Rigid Pavement Overlay (Rigid Pavement) Rp. 9,114,626.20 / segments. 
VOLUME 2 | NUMBER 2 | MARCH 2020

Available online at

http://proceedings.worldconference.id.

\section{References}

Radar Karawang. (2019, February). Damage Road Badami - Loji Increasingly Severe.

IDN Times, K. (2019). 1937.53 Kilometers along the highway in Karawang Damaged

Directorate General of Highways. (1991). Rigid Pavement Maintenance Procedures

(Concrete Road Works Planning 2003). pd T-14-2003. In planning the concrete road pavement

Ministry of Resettlement and Prasaranawilayah. (2003). Cement Concrete Pavement Planning, Ministry of Settlement and Prasaranawilayah

Rigid Pavement Maintenance Procedures (Rigid Pavement) No. 10 / T / BNKT / (1991) .Direktorat General of Highways. 